Revue d'histoire de l'Amérique française

REVUE D.HISTOIRE DE L'AMÉRIQUE FRANÇAISE

\title{
De soeur Marie-Achille à soeur Marie-Zozime
}

L'usage des prénoms masculins chez les Soeurs des Saints

Noms de Jésus et de Marie (1844-1969)

\section{Dominique Laperle}

Volume 60, numéro 3, hiver 2007

URI : https://id.erudit.org/iderudit/015963ar

DOI : https://doi.org/10.7202/015963ar

Aller au sommaire du numéro

Éditeur(s)

Institut d'histoire de l'Amérique française

ISSN

0035-2357 (imprimé)

1492-1383 (numérique)

Découvrir la revue

Citer cette note

Laperle, D. (2007). De soeur Marie-Achille à soeur Marie-Zozime : l'usage des prénoms masculins chez les Soeurs des Saints Noms de Jésus et de Marie (1844-1969). Revue d'histoire de l'Amérique française, 60(3), 355-372.

https://doi.org/10.7202/015963ar d'utilisation que vous pouvez consulter en ligne.

https://apropos.erudit.org/fr/usagers/politique-dutilisation/ 


\section{Note de recherche}

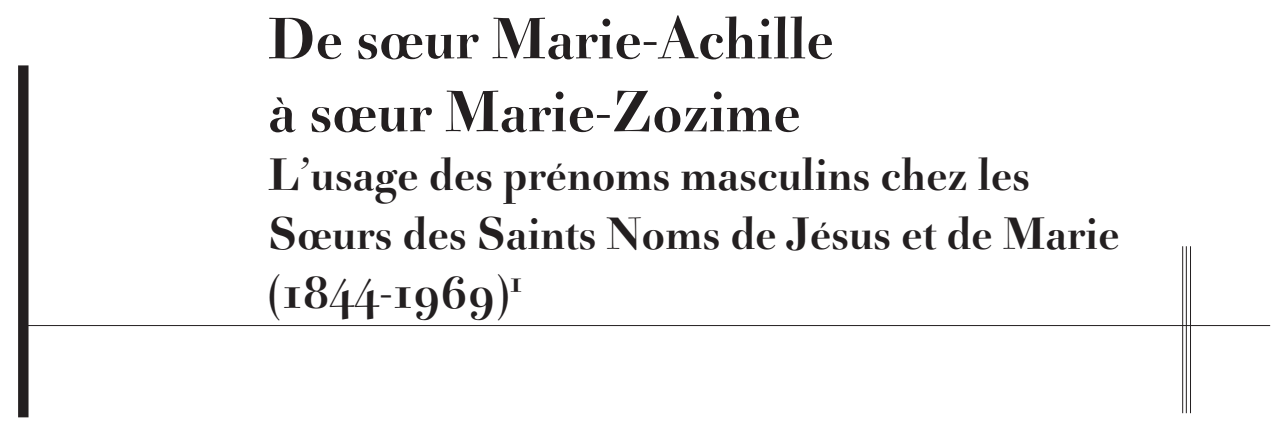

DOMINIQUe LAPERLE

Pensionnat du Saint-Nom-de-Marie

"Oui, nous les appellerons les Bonnes Sœurs même si certaines d'entre elles persistent à porter des noms redoutables².»

Dour de nombreux spécialistes de l'histoire de l'Église et des congré-
gations religieuses au Québec, la période allant de 1840 à 1960 est un véritable âge d'or. Cette perception s'explique à la fois par la multiplication des communautés, une progression constante des vocations et une diversification des fonctions ${ }^{3}$. À cet égard, les communautés féminines sont

1. Cet article a été rédigé dans le cadre du centenaire du Pensionnat du Saint-Nom-de-Marie. L'auteur tient à remercier la directrice de la revue, Christine Hudon, les lecteurs anonymes et le comité de rédaction pour leurs commentaires sur cet article. Nos remerciements vont aussi à Lucia Ferretti, Pietro Boglioni et Denise Bourgeois pour leurs précieux conseils. Enfin, cet article n’aurait pu voir le jour sans l'aide précieuse des archivistes Yvonne Painchaud et sœur Lucille Potvin, snjm. Outre les documents ayant permis la constitution du corpus du personnel religieux, elles ont commenté de nombreuses versions préalables de ce texte. Je leur dédie cet article.

2. Benoît Lacroix, La foi de ma mère. La religion de mon père (Montréal, Bellarmin, 2003), 256.

3. Sur les dimensions sociologiques des vocations, voir Diane Bélanger et Lucie Rozon, Les religieuses au Québec (Montréal, Libre Expression, 1982); Marta Danylewycz, Profession : religieuse. Un choix pour les Québécoises (Montréal, Boréal, 1988); Nadia-F. Eid et Micheline Dumont, Maîtresses de maison, maîtresses d'école. Femmes, familles et éducation dans l'histoire du Québec (Montréal, Boréal Express, 1983); Marguerite Jean, Évolution des communautés religieuses de femmes au Canada de 1639 à nos jours (Montréal, Fides, 1997); Nicole Laurin, Danielle Juteau et Lorraine Duchesne, À la recherche d'un monde oublié. Les communautés religieuses de femmes au Québec de 1900 à 1970 (Montréal, Le Jour Éditeur, 1991); Danielle Juteau et Nicole Laurin, Un métier et une vocation. 
particulièrement dynamiques. Pour plusieurs, outre la dimension spirituelle, ce phénomène trouve ses racines dans le conservatisme et la domination masculine de la société québécoise d’alors. En effet, les études de genre récentes démontrent qu'au $\mathrm{XIX}^{\mathrm{e}}$ siècle, les femmes subissent une incessante subordination sur le plan du travail, une exclusion sur le plan politique (retrait du droit de vote en 1849) ainsi qu'une «infantilisation légale» face au père ou à l'époux (Code civil de 1866). En plus de tout cela, les études supérieures leur sont, à toutes fins utiles, bloquées; afin de "respecter leur nature», les femmes sont maintenues dans des programmes scolaires s'articulant sur les «fonctions traditionnelles», c'est-àdire les travaux d'aiguille, les arts culinaires et ménagers. L’entrée en communauté permettait donc à plusieurs jeunes femmes de se détacher d'un destin par trop contraignant, afin de s'inscrire dans un cheminement qui assurait une formation intellectuelle, des carrières variées, un épanouissement artistique et la possibilité de voyager en pays de mission. Ultimement, on évitait un célibat laïque parfois mal perçu dans la société globale ${ }^{4}$ et on remplaçait le modèle traditionnel de la procréation par une forme de "maternité spirituelle ${ }^{5}$. Ainsi, l'adhésion aux communautés religieuses a permis à plusieurs femmes «d'établir, consolider et élargir un espace propre d'intervention sociale ${ }^{6} »$ : autrement dit, de se construire une identité.

Dans un ouvrage de 1995, Micheline Dumont pose l'hypothèse de la vocation religieuse comme "une avenue d'égalité avec les hommes [autorisant] l'exercice de fonctions interdites aux femmes ${ }^{7}$ ». Cet égalitarisme se traduirait aussi par la prise de noms d'hommes par $40 \%$ des sœurs, question de "contrer l'image sociale de la féminité et d'autoriser les responsabilités dites masculines de tant de religieuses ${ }^{8} »$. Cette dernière

Le travail des religieuses au Québec de 1901 à 1971 (Montréal, Presses de l'Université de Montréal, 1997).

4. Catherine Renaud, «Une place à soi? Aspects du célibat féminin laïc à Montréal à la fin du XIX ${ }^{\mathrm{e}}$ siècle», mémoire de maîtrise (histoire), Université de Montréal, 1993. Voir aussi Elizabeth Abbot, Histoire universelle de la chasteté et du célibat (Montréal, Fides, 2001); Jean-Claude Bologne, Histoire du célibat et des célibataires (Paris, Fayard, 2004).

5. Martine Spielvogel, «La maternité spirituelle: une analyse du discours sur la vocation religieuse féminine», dans Louise Vandelac et al., Du privé au politique : la maternité et le travail des femmes comme enjeux des rapports de sexe (Montréal, Université du Québec à Montréal, "Actes de la session d'études féministes du Congrès de l'ACFAS 1989», 1990), 44.

6. Yolande Cohen, «La critique et ses dessous », Clio, 6 (1997): [en ligne], http:/ / clio.revues. org, consulté le 10 mai 2006.

7. Micheline Dumont, Les religieuses sont-elles féministes? (Montréal, Bellarmin, 1995), 40.

8. Ibid., 41. 
assertion mérite que l'on s'y arrête, car elle soulève l'idée que la masculinisation symbolique du nom de la religieuse lui conférerait une plus grande individualité et lui permettrait un épanouissement professionnel ${ }^{9}$. Qu'en est-il vraiment? Un usage généralisé de la dénomination masculine suggérerait-il une volonté d' "asexualisation » ou d'indifférenciation? Cela pourrait confirmer la thèse féministe d'une appropriation masculine de l'organisation et de la symbolique religieuses ${ }^{10}$.

Cette question ne semble pas avoir attiré beaucoup l'attention des chercheurs. Les études des dernières années portent souvent sur le travail spécifique des congrégations en éducation ${ }^{11}$, en santé et dans le domaine social $^{12}$. La question de la féminité des religieuses a aussi été abordée, notamment dans des études analysant la symbolique de leur costume, mais l'usage et la signification de la dénomination, eux, n'ont pas fait l'objet d'études approfondies ${ }^{13}$. Le présent article essaie donc de combler un vide. Nous chercherons d'abord à comprendre le mécanisme de l'attribution des noms (modalités, influences spirituelles et familiales). Ensuite, nous identifierons le sous-groupe composé des sœurs portant un prénom masculin. Ces noms seront compilés et ventilés par périodes afin d'en vérifier la constance ou les particularités.

Cette analyse porte spécifiquement sur les Sœurs des Saints Noms de Jésus et de Marie. Il s'agit d'une communauté enseignante fondée à Longueuil en 1843 par mère Marie-Rose (Eulalie Durocher), à l'instigation de $\mathrm{M}^{\mathrm{gr}}$ Ignace Bourget, évêque de Montréal ${ }^{14}$. Cette congrégation, de

9. Elizabeth Dufourcq, Les congrégations religieuses féminines hors d'Europe (Paris, Librairie de l'Inde, 4 tomes, 1993), $1: 52$.

10. Denise Veillette, "Hiérarchisation sociale des sexes, occupations des femmes et appropriation masculine du sacré », dans Denise Veillette, dir., Femmes et religion (Sainte-Foy, Les Presses de l’Université Laval, 1995), 16.

11. Josée Desbiens, Le collège Regina Assumpta (1955-1995): 40 ans d'éducation au féminin (Montréal, Fides, 1995); Carole Gélinas, La fabuleuse histoire d'une école: Villa Maria (Montréal, Villa Maria, 2004); Dominique Laperle, Il y a cent ans que nous pensons à l'avenir. Histoire du Pensionnat du Saint-Nom-de-Marie, 1905-2005 (Montréal, Comité du centenaire du Saint-Nom-deMarie, 2005).

12. François Rousseau, La croix et le scalpel. Histoire des Augustines et de l'Hôtel-Dieu de Québec (Sillery, Septentrion, 2 tomes, 1989 et 1994); Nive Voisine et Yvonne Ward, Histoire des Sours de la charité de Québec (Beauport, MNH, 3 tomes, 1998 et 2003).

13. Voir Kristoff Talin, "Les religieuses, l’Église catholique et le monde moderne», dans Françoise Lautman, dir., Ni Ève ni Marie. Luttes et incertitudes des héritières de la Bible (Genève, Labor et Fides, 1997), 157-180.

14. Pour mieux connaître la fondatrice et la communauté, voir Germaine Duval, snjm, Par le chemin du Roi, une femme est venue (Montréal, Bellarmin, 1982). 
droit pontifical depuis $1877^{15}$, va connaître une des plus fortes croissances de l'époque, passant de 30 professes en 1849 à 4211 religieuses en $1966^{16}$. Les premières fondations de la communauté basée d'abord à Longueuil (maison mère et pensionnat en 1844) se font dans la vallée laurentienne (Belœil, 1846; Saint-Lin et Saint-Timothée, 1848). Par la suite, la communauté concentre l'essentiel de ses activités sur l'île de Montréal et ses environs ${ }^{17}$ et y ouvre de nombreux établissements (maison mère, noviciat et pensionnat à Hochelaga en 1860, l'Académie Marie-Rose en 1876, le Pensionnat Mont-Royal en 1898, le Pensionnat du Saint-Nom-de-Marie à Outremont en 1905 et la nouvelle maison mère, dans la même municipalité, en 1925). La congrégation essaime aussi en «terres de mission»; d'abord en Oregon en 1859, en Californie et en Floride en 1868, puis, ailleurs au Canada, en Afrique et en Asie ${ }^{18}$. Du jardin d'enfants au cours primaire public et privé, en passant par des écoles normales, ménagères, de musique ou même des universités, l'œuvre éducative des Sœurs des Saints Noms de Jésus et de Marie s'étend à tous les niveaux de la formation des jeunes, surtout des filles.

Notre étude couvre la période allant de 1844, année des vœux de la fondatrice, à 1969, date où, dans la foulée de Vatican II et du décret Perfectce Caritatis de 1965 sur l'adaptation et le renouveau de la vie religieuse, la congrégation abandonne, à toutes fins utiles, la tradition de donner un nouveau nom aux professes. Nous avons eu recours au répertoire informatisé des religieuses, disponible au Service central des archives des Sœurs des Saints Noms de Jésus et de Marie (SCA SNJM). Cette base de données Access comprend, entre autres, le nom civil de la religieuse, la date des premiers vœux ainsi que le nom et le prénom du père. Notre corpus de départ comporte les noms des 6964 religieuses ayant prononcé des vœux $^{19}$. Il exclut celles qui n’ont pas dépassé l'étape du postulat. Nous

15. Sur les distinctions entre une communauté diocésaine et pontificale, voir M. Jean, Évolution des communautés religieuses de femmes..., op. cit., particulièrement la deuxième partie portant sur le développement juridique des communautés et le chapitre 15, Recours aux approbations romaines (1860), 221-233.

16. G. Duval, snjm, Par le chemin du Roi..., op. cit., 379.

17. M. Dumont, Les couventines..., op. cit., 262.

18. René Duchaussois, omi, Rose du Canada (Montréal, Granger \& Frère, 1932), 343.

19. Chez les SNJM, le postulat durait généralement six mois. Il était suivi d'une première année dite de noviciat canonique. Après cette première année, la novice quittait la maison mère et allait travailler durant six mois dans une des maisons de la communauté (noviciat-missionnaire). Ensuite, suivait l'étape des premiers vœux (pauvreté, chasteté, obéissance) de trois ans, suivis des seconds (une année plus tard), des troisièmes vœux (un an de plus) et, enfin, des vœux perpétuels. Voir aussi N. Laurin, D. Juteau et L. Duchesne, À la recherche d'un monde oublié..., op. cit., 270. 
avons écarté de ce corpus initial les six cas de religieuses n'ayant reçu, comme prénom usuel, qu'un suffixe religieux contenant le terme "Marie» (par exemple, Du Saint-Nom-de-Marie, Du Saint-Cœur-de-Marie) et n'avons pas non plus considéré tous les suffixes introduits par une particule (par exemple, de l'Eucharistie, of the Cross) qui suivaient un prénom. Au final, ce sont 6958 des 6964 noms de religieuses qui ont été analysés.

Pour compléter ces informations nominatives, nous avons procédé à une série d'entrevues, en décembre 2005, avec des religieuses œuvrant au Pensionnat du Saint-Nom-de-Marie et à l'École de Musique Vincentd'Indy. Ces rencontres visaient à connaître les raisons ayant motivé le choix du prénom et à cerner les impressions des religieuses face à sa symbolique et son usage. Pour les fins de notre étude, nous utiliserons indifféremment les termes religieuses, sœurs ou professes. Pour alléger le texte, la Congrégation des Sours des Saints Noms de Jésus et de Marie sera représentée par le sigle SNJM.

\section{LA PRÉNOMINATION: USAGES SOCIAUX, USAGES RELIGIEUX}

Dans la société globale, le prénom a comme fonctions d'identifier la personne et de préciser le sexe de celle qui le porte. Il peut aussi devenir important sur le plan du patrimoine symbolique et de la reproduction sociale avec sa transmission à la génération suivante ${ }^{20}$. Dès l'époque de la Nouvelle-France, l'Église catholique cherche à s'assurer que les nouveaunés, à leur baptême, reçoivent le prénom d'un saint reconnu. $\mathrm{M}^{\mathrm{gr}} \mathrm{de}$ Saint-Vallier insiste fortement sur cette question dans son Rituel du Diocèse de Québec en proposant pas moins de 1624 prénoms $^{21}$. À cet effet, le XIX ${ }^{\mathrm{e}}$ siècle est plus libéral, la population du Canada français pouvant puiser dans une banque de 19000 prénoms ${ }^{22}$. Malgré cette abondance, certains prénoms sont adoptés par les hommes et les femmes, ce qui ne concorde pas avec la vision de l'Église sur la différenciation des $\operatorname{sexes}^{23}$. Certaines recherches récentes soutiennent que l'usage de prénoms homonymes pour les deux sexes amoindrit les frontières sociales entre les hommes et

20. Louis Duchesne, Les prénoms, des plus rares aux plus courants au Québec (Outremont, Trécarré, 2001), 10; Geneviève Ribordy, Les prénoms de nos ancêtres (Sillery, Septentrion, 1995), 11.

21. Rituel du diocèse de Québec publié par ordre de $M^{\text {gr }}$ de Saint Vallier, évêque de Québec (Paris, S. Langlois, 1703).

22. Jean-Claude Hurieux, Le portail des prénoms de la francophonie [en ligne] http: / / cafe.rapidus. net/jhuriaux/index.html, consulté le 19 décembre 2005.

23. Denise Veillette, «Hiérarchisation sociale des sexes...», loc. cit., 16. 
les femmes et permet à ces dernières d'agir davantage de manière masculine ${ }^{24}$. L'attribution de prénoms masculins n'est donc pas un phénomène propre aux religieuses. Geneviève Ribordy écrit d'ailleurs qu'au $\mathrm{xVIII}^{\mathrm{e}}$ siècle, il atteint, selon les catégories sociales, de $3 \%$ à $5 \%$ des filles nées de mariages contractés entre 1700 et $1729^{25}$. Cette idée se perçoit-elle dans l'attribution des noms masculins ou homonymes chez les religieuses?

Dans les communautés religieuses, la construction d'une nouvelle identité sociale avec le changement de nom, le jour de la vêture, existe depuis le Haut Moyen Âge ${ }^{26}$. Dans la tradition biblique du rite de passage, le changement de nom témoigne d'une union mystique avec Dieu. C'est habituellement au moment où "elle meurt au monde ${ }^{27}$ " et rend grâce à Dieu (Deo gratias), que la jeune postulante reçoit son habit et sa nouvelle identité. Cette tradition n'est, par contre, pas présente ou appliquée de la même façon dans toutes les congrégations.

Ainsi, les premières Sœurs de la Congrégation de Notre-Dame signaient leur nom civil et religieux (par exemple, Marguerite Bourgeoys dite Sœur du Saint-Sacrement). Au xvII ${ }^{\mathrm{e}}$ siècle, certaines professes ne reçurent pas de noms religieux. Puis le nom civil disparut complètement pour faire place au nom de religion et, par tradition, la Congrégation n'utilisa plus que le nom d'un saint ou d'une sainte (par exemple, Marie-Ursule Labelle devint Sœur Sainte-Ursule). La future novice pouvait exprimer ses préfé-

24. «When women use men's names, we have a situation in which a lower-status group is borrowing a symbol or accoutrement of a higher-status group... In other expressive domains as well, women freely borrow male symbols (e.g., fashions in clothing), while men shy away from female symbols», R. D. Alford, "Naming Identity: A Cross-Cultural Study of Personal Naming Practices", Language in Society, 19 (1988): 69-143 et Stanley Lieberson et al., "The Instability of Androgynous Names: The Symbolic Maintenance of Gender Boundaries ", America Journal of Sociology, 105,5 (mars 2000): 1249-1287.

25. G. Ribordy, Les prénoms de nos ancêtres..., op. cit., 67-68.

26. M. Jean, Évolution des communautés religieuses de femmes..., op. cit., 10. Se référant à l'article «Nome di religione» dans le Dizionario degli istituti di perfezione, VI (1980): 321-325, le professeur Pietro Boglioni nous mentionnait aussi que, dans l'Antiquité, cet usage était plutôt rare et se justifiait par une raison précise, comme celle de changer un nom païen, par exemple le pape Jean II qui s'appelait auparavant Mercure. Ce premier changement serait, d'après lui, le premier cas connu et daterait de 533. L'habitude se généralise dans les familles réformées des ordres religieux, surtout après le Concile de Trente. Enfin, les situations varient selon les diverses règles. "Dans certaines congrégations d'origine française, on prit aussi l'habitude de donner, à la place du nom de baptême ou du nom de famille, le nom d'un saint, précédé justement du terme «saint» (par ex. : "sœur Saint-Joseph»)».

27. Yvonne Turin, Femmes et religieuses au XIX $x^{\mathrm{e}}$ siècle (Paris, Nouvelle cité, 1989), 205. 
rences au sujet de son nom de religion, mais le Conseil général avait le dernier $\operatorname{mot}^{28}$.

La majorité des Sœurs Grises gardaient quant à elles leur nom propre et étaient désignées par leur nom de famille (par exemple, sœur Despins). Lorsque plusieurs religieuses avaient le même nom de famille simultanément, on les nommait par leur prénom et leur nom (par exemple, sœur Blanche Boisvert était nommée sœur Boisvert, tandis que sœur Madeleine Boisvert, plus jeune, était nommée sœur Madeleine Boisvert). Pour se distinguer de ses homonymes, une religieuse pouvait aussi prendre le nom de famille de sa mère. Si plusieurs sœurs d'une même famille entraient en religion, on leur donnait un nom religieux (par exemple, sœur SainteThérèse-de-l'Enfant-Jésus). L'attribution d'un nom religieux n'obéissait pas à des règles précises, car rien n'est évoqué à ce sujet dans les Coutumes et Règles des Soeurs de la Charité de Montréal. À partir de 1915 environ, les religieuses n'utilisèrent plus de noms religieux ${ }^{29}$.

Chez les Sœurs de Sainte-Anne, une communauté créée à Vaudreuil en 1850 sous l'épiscopat de $\mathrm{M}^{\mathrm{gr}}$ Bourget, les constitutions de 1927 indiquent que la Supérieure générale "assignera à chaque sœur le nom qu'elle doit porter en religion ». Dans cette communauté, les sœurs soumettaient trois noms, mais il arrivait que la Supérieure en choisisse un autre. Le préfixe Marie était toujours donné (par exemple, Sœur Marie-Lambert, Sœur Marie-Walter) ${ }^{30}$.

Enfin, chez les Dominicaines du Rosaire de Trois-Rivières, une petite communauté fondée en 1902, des motivations très diverses guidaient le choix des noms. Certaines prenaient par exemple le nom d'une professe décédée quelques jours avant leur prise d'habit. Le désir d'honorer un saint, une sainte ou une figure importante de la tradition dominicaine (par exemple, Sour Saint-Thomas-d'Aquin, Sour Catherine-de-Sienne) ou celui de rappeler le nom d'un curé ou d'un prêtre ayant favorisé la vocation pouvait induire certains choix. Parfois, la jeune postulante choisissait elle-même son nom de religieuse. Certaines ont ainsi ajouté un prénom à celui de leur père ou de leur mère. Bref, il n’y avait pas de règles précises.

28. Je remercie sœur Raymonde Sylvain, cnd, archiviste de la Congrégation Notre-Dame, pour ces informations.

29. Je remercie Mylène Laurendeau, archiviste des Sœurs de la Charité de Montréal, pour ces informations.

30. Je remercie Hélène Élément, archiviste de la Congrégation des Sœurs de Sainte-Anne pour ces informations. 
Par ailleurs, l'usage de prénoms masculins était plutôt restreint, sauf Dominique et ses composés (par exemple, Dominique-de-la-Croix) ${ }^{31}$.

Ce survol des modalités d'attribution des noms dans différentes congrégations religieuses féminines permet de dégager une impression générale: l'attribution des noms d'hommes n'existe tout simplement pas dans certaines communautés ou représente une manifestation mineure dans d'autres. Les communautés apparues sous l'épiscopat de $\mathrm{M}^{\mathrm{gr}}$ Bourget c'est le cas des Sœurs de Sainte-Anne et, comme nous le verrons, des SNJM - semblent donc, plus touchées par ce phénomène. Qu'en est-il du clergé et des religieux masculins? Les prêtres séculiers et la plupart des membres des congrégations dites régulières conservaient leur nom laïque. Le phénomène du changement de noms est surtout perceptible chez les ordres contemplatifs et certaines congrégations de frères. Contrairement aux femmes, les hommes ne prennent pas de prénoms religieux de l'autre sexe, sauf Marie (par exemple, Conrad Kirouack est devenue le célèbre frère Marie-Victorin).

\section{LES NOMS CHEZ LES SNJM}

Chez les SNJM, l'attribution du nom de la fondatrice par $\mathrm{M}^{\mathrm{gr}}$ Ignace Bourget lance, en quelque sorte, l'histoire des prénoms dans cette communauté. En février 1844, lors de la cérémonie des vœux d'Eulalie Durocher et de deux compagnes, il déclarait: «Désormais vous ne vous appellerez plus demoiselle Henriette Céré, mais sœur Marie-Madeleine. Désormais vous ne vous appellerez plus demoiselle Marie-Josèphe Dufresne, mais sœur Marie-Agnès. Désormais vous ne vous appellerez plus demoiselle Mélanie-Eulalie Durocher, mais sœur Marie-Rose ${ }^{32}$.»

Bourget et les différents prêtres proches de la communauté ont-ils pu influencer le choix des prénoms? Rien dans nos recherches ne le confirme. Quel est le degré réel de liberté des religieuses dans ce choix? Les archives des SNJM sont avares de documents qui décrivent le processus suivi pour l'attribution des noms des professes. Selon la tradition orale, la procédure diffère selon les époques. À certains moments, la postulante inscrivait les prénoms de tous les membres de sa famille (père, mère, frères et sœurs). À d'autres, elle ne soumettait que trois noms par écrit à la secrétaire générale. Les entrevues menées auprès de plusieurs religieuses permettent

31. Je remercie Lucia Ferretti pour ces informations. Voir son livre "C'est à Moi que vous l'avez fait». Histoire des Dominicaines de Trois-Rivières (Sillery, Septentrion, 2002), 68.

32. G. Duval, snjm, Parle chemin du Roi..., op. cit., 170. 
de confirmer que les noms soumis sont porteurs d'une charge affective importante comme de "l'admiration" ou un "amour profond». Pour d'autres, le choix reposait, plus simplement, sur une «belle sonorité». Cela dit, une jeune religieuse qui «désirait trop ardemment un nom » ne l'obtenait à peu près jamais, et ce, pour inculquer l'humilité et la soumission.

Rien dans les annales des SNJM ne confirme si la secrétaire générale prenait la décision seule. Il est probable que les autres membres du Conseil général étaient à tout le moins informées du choix, compte tenu de la charge symbolique des prénoms attribués. À titre d'exemple, Mère MarieRose se serait exclamée au Conseil à propos d'une postulante: «Donnonslui un nom d'ange car elle en a toutes les vertus ${ }^{33}$.» Il appert que les religieuses se référaient à des ouvrages reconnus par l'Église pour le choix du nouveau prénom ${ }^{34}$. Les SNJM ont rejeté, dès le départ, les suffixes à consonance doloriste (des Sept plaies, du Christ Agonisant) ou fondamentalistes (du Divin-Tabernacle, de la Sainte-Face) en usage dans certaines congrégations. Enfin, la secrétaire générale pouvait s’inspirer d'événements circonstanciels (béatification d'un saint, année mariale de 1954) ou, plus simplement, du nom de la paroisse d'origine de la postulante. Pour ce dernier exemple, une religieuse nous racontait lors d'une entrevue:

Je désirais, comme nom religieux, Marie de la joie. La secrétaire générale me fit comprendre que les événements de la vie allaient parfois me rendre ce nom bien lourd à porter. D’autre part, certaines rimes faciles pouvaient «dénaturer» la dimension sacrée du nom. Finalement, j’ai accepté sa suggestion de porter le nom de ma paroisse d'origine, qui sonnait plutôt bien ${ }^{35}$.

Chez les SNJM, toutes les professes de la congrégation reçoivent le prénom «Marie». Pour 155 professes ( $2 \%$ du corpus), c’est le seul prénom porté (par exemple, Marie de la Croix). Pour d'autres, Marie est l'un des deux éléments d'un prénom composé. Il se retrouve tantôt au début (Marie-Stéphane), tantôt à la fin (Valentine-Marie). Dans ces conditions, le prénom «Marie» est couramment employé et fait pleinement partie de l'identité des religieuses.

33. R. Duchaussois, omi, Rose du Canada..., op. cit., 215.

34. Dans les archives des SNJM, on retrouve ainsi une Vies des Pères, des martyrs et des autres principaux saints, tirées des Actes originaux \& des monuments les plus authentiques (Paris, Pierre Vedeilhié,1763), 12 vol. Aussi: $\mathrm{M}^{\mathrm{gr}}$ Paul Guérin, Les Petits Bollandistes. Vie des saints de l'Ancien et du Nouveau Testament (Paris, Bloud et Barral, [s.d.]), 18 volumes. Il s'agit vraisemblablement d'un tirage qui précède l'édition de 1884 .

35. Entrevue accordée sous le couvert de l'anonymat. 
Dans d'autres cas, le prénom «Marie» est plus emblématique. Il précède un prénom simple suivi d'une particule et d'un suffixe de type religieux (Marie Denyse de la Croix, Marie Claire du Rédempteur, Marie Julien du Saint-Sacrement). On le retrouve aussi devant un prénom composé entièrement féminin (Marie Cécile-Romaine, Marie Louise-Anne, Marie DeniseAntoinette) ou masculin (Marie Pierre-Yvan, Marie Robert-Joseph, Marie Marcel-Alphonse). Enfin, il prélude le nom complet d'un saint ou d'une sainte (Marie Thomas-d'Aquin, Marie Thérèse-de-l'Enfant-Jésus), Pour tous ces exemples, la religieuse écrit toujours l'initiale du «Marie» dans ses documents officiels mais ne l'emploie que rarement dans la langue parlée (par exemple, Sœur Marie Jean-Gualbert signera toujours «M. JeanGualbert» mais se fera appeler "sœur Jean-Gualbert», sœur Marie Irène de la Trinité signera «M. Irène de la Trinité», mais se fera appeler «sœur Irène de la Trinité», voire "sœur Irène»). On le constate donc, pour ces dernières classes de prénoms, l'usage de «Marie» est davantage spirituel qu'usuel. D’où notre décision de ne pas en tenir compte dans notre analyse de ces formes particulières de noms.

Plus de 1255 sœurs, soit $18 \%$ de la congrégation étudiée, portent par ailleurs un prénom qui assemble des éléments de genre différent (MarieMénodore, Marie-Tarcisius, Vincent-Marie). Pour faire une analyse approfondie des prénoms composés chez les religieuses du SNJM, nous avons créé deux catégories tenant compte de l'ordre hiérarchique du genre des prénoms: l'une d'elles regroupe les prénoms composés commençant par un élément masculin ( $\mathrm{dm}$ ) (Louis-Marie, Aurèle-Marie); l'autre, les prénoms composés commençant par un prénom féminin (df) (MarieEthelbert, Marian-Bruce).

Dans le cas des prénoms homonymes indistinctement attribués aux hommes et aux femmes (Camille, Claude, Carol, Dominique, etc.), nous avons choisi de respecter le sexe du porteur original, saint ou apôtre, tel que reconnu, sauf si l'orthographe du prénom permet un classement clair vers le genre féminin (par exemple, Andrée au lieu d'André).

Le classement décennal des prénoms selon le genre (tableau 1) permet de dégager certaines tendances. Les vingt premières années d'existence de la communauté sont marquées par un usage restreint des prénoms masculins. De 1844 à 1863, 67\% des religieuses utilisaient un prénom totalement féminin et $27 \%$ en avaient un qui l'était partiellement. La pratique se transforme après 1864 , alors que l'usage de prénoms masculins tend à augmenter. Entre 1904 et 1963, les prénoms totalement masculins 
se maintiennent autour de $23 \%$, atteignant un sommet de $26 \%$ entre 1914 et 1923 . Après 1963 , ce nombre tombe sous la barre des $20 \%$.

Tableau I

Répartition des prénoms selon le genre par décennie d'entrée

\begin{tabular}{|c|c|c|c|c|c|c|c|c|c|}
\hline DATES & $\mathrm{Ma}^{1}$ & $\%$ & $\mathrm{Fe}^{2}$ & $\%$ & $\mathrm{Dm}^{3}$ & $\%$ & $\mathrm{Df}^{4}$ & $\%$ & Total \\
\hline $1844-1853$ & 4 & 7 & 40 & 68 & - & - & 15 & 25 & 59 \\
\hline $1854-1863$ & 5 & 5 & 67 & 66 & - & - & 30 & 29 & 102 \\
\hline $1864-1873$ & 23 & 12 & 91 & 47 & - & - & 79 & 41 & 193 \\
\hline $1874-1883$ & 16 & 7 & 114 & 50 & - & - & 97 & 43 & 227 \\
\hline $1884-1893$ & 39 & 13 & 157 & 51 & 1 & 0,3 & 108 & 35 & 305 \\
\hline 1894-1903 & 64 & 17 & 213 & 55 & 6 & 2 & 103 & 27 & 386 \\
\hline 1904-1913 & 135 & 20 & 409 & 60 & 13 & 2 & 123 & 18 & 680 \\
\hline $1914-1923$ & 218 & 26 & 531 & 63 & 25 & 3 & 68 & 8 & 842 \\
\hline 1924-1933 & 264 & 23 & 732 & 65 & 69 & 6 & 66 & 5 & 1131 \\
\hline 1934-1943 & 202 & 22 & 590 & 64 & 53 & 6 & 70 & 4 & 915 \\
\hline 1944-1953 & 167 & 21 & 504 & 64 & 54 & 7 & 68 & 9 & 793 \\
\hline 1954-1963 & 223 & 24 & 567 & 62 & 67 & 7 & 64 & 7 & 921 \\
\hline 1964-1969 & 78 & 19 & 250 & 62 & 40 & 10 & 36 & 9 & 404 \\
\hline Total & 1438 & 21 & 4265 & 61 & 328 & 5 & 927 & 13 & 6958 \\
\hline
\end{tabular}

Source: Base de données Access SCA SNJM.

1 Ma: Prénom entièrement masculin excluant Marie.

2 Fe: Prénom entièrement féminin.

$3 \mathrm{Dm}$ : Prénom composé commençant par un terme masculin mais associé à Marie ou un de ses composés.

4 Df: Prénom composé commençant par Marie ou un de ses composés et suivi d'un terme masculin.

La catégorie des prénoms composés commençant par un élément masculin est inexistante pendant les cinquante premières années. Ce type de désignation fait une timide apparition à la fin du $\mathrm{XIX}^{\mathrm{e}}$ siècle, prend son envol à partir de 1924 et augmente graduellement pour atteindre son sommet de $10 \%$ dans la dernière décennie étudiée.

La catégorie des prénoms composés commençant par un terme féminin connaît des résultats inégaux. Entre 1844 et 1863, c’est plus du quart des effectifs qui recourent à ce type d'association. Dans les deux décennies suivantes, soit de 1864 à 1883 , il atteint $42 \%$ de la prénomination. Par la suite, il décroît de manière continue pour s'établir à $4 \%$ en 1934-1943) et, dès 1944, reprend une courbe ascendante jusqu'en 1969 pour terminer à $9 \%$ chez les professes. 
Il est difficile de confronter nos résultats à ceux de Micheline Dumont, car celle-ci ne discrimine pas les noms entièrement masculins de ceux qui ne le sont que partiellement. Néanmoins, la proportion de SNJM utilisant un nom complètement masculin est bien en deçà du $40 \%$ qu'elle présentait. En fait, on peut se demander si ce n'est pas l'augmentation du nombre de vocations qui en a provoqué un usage plus important. La liste des saints masculins étant plus dense, il faut peut-être voir ici des considérations pratiques plutôt qu'une volonté d'asexualisation des sœurs. Enfin, même si certains assemblages de prénoms peuvent paraître pittoresques, il faut se rappeler qu'ils puisaient généralement dans la liste soumise par la postulante.

\section{LES PRÉNOMS MASCULINS}

Pour mieux comprendre l'usage des prénoms masculins, nous les avons isolés. Au total, ce sont 523 appellations masculines différentes qui ont été utilisées pour prénommer les SNMJ. Trente-cinq de ces prénoms suffisent pour identifier $70 \%$ des religieuses utilisant un prénom masculin (tableau 2). Jean/John remporte la palme avec plus de $11 \%$ de toutes les attributions masculines. L'usage de ce prénom chez les SNJM diffère des pratiques observées dans la population en général. En effet, Jean a été, tout au long de l'histoire de la Nouvelle-France, un prénom important ${ }^{36}$. Son usage s'estompe un peu au XIx ${ }^{\mathrm{e}}$ siècle et revient, à partir des années 1920, surtout comme principal élément des prénoms composés. Or, à l'échelle globale, il ne dépasse jamais la barre des $3 \%$. Joseph, comme deuxième prénom le plus courant chez les $\mathrm{SNJM}$, n'est pas une surprise. À titre de patron du Canada, on vénère beaucoup ce saint. Il occupe la première place des prénoms les plus donnés, de 1880 à 1909, dans la société civile ${ }^{37}$. Sa popularité à l'intérieur de la communauté s'explique aussi par le fait qu'on le considère comme le premier économe de l'Institut et que son intercession est précieuse dans les affaires financières. Les trois prénoms suivants, Pierre, Louis et François, obtiennent des résultats surprenants qui s'apparentent davantage à ceux de Geneviève Ribordy pour l'époque de la Nouvelle-France. C'est comme si la communauté puisait aux sources de l'histoire, alors que les prénoms masculins utilisés dans la société globale se renouvellent plus rapidement.

36. Ibid., 140. Lire en particulier la note concernant le prénom composé Jean-Baptiste, l’un des plus populaires de cette époque.

37. L. Duchesne, Les prénoms..., op. cit., 17. 
Tableau 2

Principaux prénoms masculins

$\begin{array}{lcl}\text { Prénom } & \text { Occurrences } & \text { Prénom } \\ \text { Jean/John } & 180 & \text { André/Andrew } \\ \text { Josef/Joseph } & 104 & \text { Albert } \\ \text { Pierre/Peter } & 76 & \text { Raymond } \\ \text { Louis } & 74 & \text { Gabriel } \\ \text { François/Francis } & 72 & \text { Marcel } \\ \text { Paul } & 66 & \text { Claude } \\ \text { Charles } & 48 & \text { Laurent/Lawrence } \\ \text { Michel/Michael } & 37 & \text { Eugène } \\ \text { Jacques/James } & 35 & \text { Guillaume/William/Wilhelm } \\ \text { George/s } & 33 & \text { Stanislas } \\ \text { Henry } & 27 & \text { Vincent/ius } \\ \text { Philippe } & 27 & \text { Clément } \\ \text { Antoine/Anthony } & 24 & \text { Denis/Dennis } \\ \text { Thomas } & 23 & \text { Félix } \\ \text { Bernard/Bernardus } & 22 & \text { Ange } \\ \text { Gérard } & 20 & \text { David } \\ \text { Alphonse/Alphonsus } & 19 & \text { Luc } \\ & & \text { Robert }\end{array}$

Occurrences 18 17 16 15 15 13 13 12 12 12 12 11 11 11 10 10 10 10

Source: Base de données Access SCA SNJM.

Tableau 3

Deuxième élément d'un prénom composé masculin

$\begin{array}{lclc}\text { Prénom } & \text { Occurrences } & \text { Prénom } & \text { Occurrences } \\ \text { Joseph/Josef } & 61 & \text { Gabriel } & 13 \\ \text { Michel/Michael } & 30 & \text { Jacques/James } & 13 \\ \text { François/Francis } & 27 & \text { Patrick/Patrice } & 12 \\ \text { André/Andrew } & 24 & \text { Henri/y } & 11 \\ \text { Albert } & 23 & \text { Gérard } & 11 \\ \text { Antoine/Anthony } & 18 & \text { Eugène } & 11 \\ \text { Bernard } & 16 & \text { René } & 10 \\ \text { Édouard/Edward } & 15 & \text { Alphonse } & 10 \\ \text { Paul } & 13 & & \end{array}$

Source: Base de données Access SCA SNJM. 
Dans les prénoms composés entièrement masculins qui n’utilisent le "Marie» que de manière symbolique, sur le plan de la signature (par exemple, sœur M. Clément-Maurice), nous voulions voir quels étaient ceux qui apparaissaient de manière récurrente, comme deuxième élément. Louis Duchesne mentionne que, «règle générale, le prénom composé est moins populaire que son suffixe seul ${ }^{38}$ ». Ce sont 138 prénoms qui ont été utilisés (tableau 3). De ce nombre, encore une fois, quelques-uns seulement recueillent plus de dix mentions. L'association de deux composantes masculines, aussi «viriles» soient-elles, laisse parfois de curieuses situations se produire. Sœur Jacques-René, compositrice, raconte une singulière anecdote à propos de son nom:

Plusieurs se demandent d'où vient mon nom de plume Rhené Jaque. Quand il s'est agi de faire éditer mes pièces musicales, Monsieur [Claude] Champagne me dit: "Il vous faut maintenant un nom de plume, surtout pas un nom de sœur ni de femme parce que vos pièces ne se vendront pas. » Peut-être avaitil raison. Comme je n'avais aucune expérience dans le domaine de la diffusion et de la publication, je lui faisais confiance. Un jour, il me dit qu'il avait trouvé mon nom de plume: «Vous inversez votre nom de religieuse, le tour est joué, avec une appellation qui n'a rien à voir avec un nom de famille. De cette manière, les gens ne sauront pas que vous êtes une femme ni une religieuse.» Cependant, je dois ajouter que ce pseudonyme m'a valu quelques aventures cocasses $^{39}$.

On peut s'étonner d'un tel changement de nom. Après tout, le prénom de la religieuse, s'il n'était pas précédé du titre «sœur», ne faisait guère féminin!

Dans le sous-groupe des prénoms composés de genres différents et commençant par un terme féminin, le premier prénom tourne, comme nous l'avons déjà dit, autour de Marie et de ses composés (Marian, Marie, Mary, Maria, Myriam, Maura, Maureen et Molia). Peut-on alors parler de banalisation du choix du prénom de départ? Rien n’est moins sûr. N'oublions pas la place spirituelle de Marie dans la communauté qui se réclame d'elle et qui se fait un point d'honneur de l'honorer ${ }^{40}$. Par ces choix, il y a là le témoignage d'une préférence institutionnelle, une affiliation identitaire majeure. Dans cette catégorie (Df), on associe néanmoins 428 prénoms masculins. Aucun n’est attribué plus de dix fois, la

38. Ibid., 41.

39. Cité dans «Rhené Jaque : les pièces pour les jeunes", La muse affiliée [en ligne] http:/ / www. scena.org/lamuse/current/rhene. html, consulté le 12 décembre 2005.

40. La devise de la Congrégation des SNJM est: "Jésus et Marie, ma force et ma gloire». 
palme allant à Patrick/Patrice. Il semble en fait que la majorité des religieuses qui prennent ce nom soient irlandaises (6 sur 10). Pour le reste, les prénoms masculins placés comme deuxième élément ne se distinguent pas outre mesure des choix précédents. C'est dans cette catégorie que l'on retrouve les prénoms masculins les plus pittoresques, surtout entre 1894 et 1923. Ils n'apparaissent, pour la plupart, qu'une ou deux fois. L'imagination semble sans limite. Marie-Achille côtoie Mary Pancratius, MarieThéothime et Marie-Zozime.

\section{L'INFLUENCE DU PÈRE}

Nous avons voulu aussi mesurer l'influence du nom du père auprès des religieuses (tableau 4). La base de données nous donne en effet cette information pour 6955 sœurs.

\section{Tableau 4}

Répartition des professes portant le prénom de leur père

\begin{tabular}{l|c|c|c|c|c|c}
\hline DATES & Ma & Fe & Df & Dm & Total & \% $^{\mathbf{1}^{2}}$ \\
\hline $1844-1853$ & - & - & 2 & - & 2 & 3,5 \\
\hline $1854-1863$ & - & 1 & 6 & - & 7 & 7 \\
\hline $1864-1873$ & 3 & - & 10 & - & 13 & 6,6 \\
\hline $1874-1883$ & 2 & - & 9 & - & 11 & 4,8 \\
\hline $1884-1893$ & 7 & 21 & 8 & - & 36 & 5,6 \\
\hline $1894-1903$ & 24 & 4 & 9 & 1 & 38 & 10 \\
\hline $1904-1913$ & 54 & 9 & 24 & 2 & 89 & 23 \\
\hline $1914-1923$ & 97 & 10 & 27 & 11 & 145 & 17 \\
\hline $1924-1933$ & 131 & 23 & 22 & 29 & 205 & 18 \\
\hline $1934-1943$ & 128 & 30 & 13 & 22 & 193 & 21 \\
\hline $1944-1953$ & 89 & 21 & 4 & 15 & 129 & 16 \\
\hline $1954-1963$ & 94 & 20 & 11 & 19 & 144 & 16 \\
\hline $1964-1969$ & 33 & 17 & 4 & 13 & 67 & 17 \\
\hline Total & 662 & 156 & 149 & 112 & 1079 & 15,3 \\
\hline
\end{tabular}

Source: Base de données Access SCA SNJM.

1. Le pourcentage final du tableau représente la proportion de religieuses portant le prénom du père par rapport au corpus complet.

Chez les SNJM, 1079 religieuses, soit 15,5\% du corpus total, utilisent ou s'inspirent du prénom de leur père. De ce nombre, 156 sont des féminisations du nom de baptême paternel. Outre des féminisations traditionnelles (Julie pour Jules, Jeanne pour Jean), on retrouve des variations 
excentriques. Mentionnons, entre autres, Alfredine (Alfred), Alexina (Alexis), Arsèna (Arsène), Davidica (David), Firmina (Firmin), Léonarda (Léo), Onésima (Onésime), Richardine (Richard) et Ubaldine (Ubald). Parmi les 923 autres professes, $71 \%$ d'entre elles utilisent la dénomination comme premier élément d'un prénom composé, alors que $14 \%$ du corpus des sœurs le place comme second élément du nom composé. Peu courant jusqu'en 1893, l'usage du prénom du père se multiplie après 1894. Il atteint $23 \%$ de 1904 à 1913 et $21 \%$ de 1934 à 1943 . De 1904 à 1969, il se maintient légèrement au-dessus de la moyenne de toute la période. Ici aussi, on remarque, dans les premières années d'existence de la congrégation, de 1844 à 1853 , une relative absence de cet usage, qui ne diffère pas trop de celui de la société civile où moins de $3,5 \%$ des filles de cette époque reçoivent le prénom de leur père. Par la suite, la croissance de cette pratique suit proportionnellement la courbe de l'usage des prénoms masculins. Néanmoins, on ne peut pas dire que la place du père se détache nettement. Il aurait été intéressant de pousser la comparaison des prénoms avec d'autres membres de la famille comme les frères, les grands-pères ou même les mères. Pour y arriver, nous aurions dû entreprendre une vaste recherche généalogique complémentaire, mais son ampleur nous y a fait renoncer. Il aurait aussi été intéressant de voir si des confesseurs, des mentors spirituels ou des évêques ont influencé la nomination, mais ces informations n'étaient pas accessibles ${ }^{41}$.

Toutes ces données nous ramènent à notre question de départ sur l'asexualisation ou l'indifférenciation des genres. Les religieuses, quel que soit leur prénom, sont des femmes et, au sein du christianisme, la distinction fondée sur le sexe est très marquée, ne serait-ce qu'à travers leurs rôles spécifiques en éducation, en santé et dans le service du clergé. Cependant, les entrevues réalisées auprès des religieuses démontrent qu'à leurs yeux, le prénom masculin ne confère nullement à celle qui le porte des attributs associés à la virilité. Il ne créé pas non plus de sentiment de supériorité chez les professes et il n'autorise pas l'exercice de fonctions de direction ou de gestion.

Lors des entrevues, nous voulions aussi connaître les réactions des élèves face aux noms religieux. La plupart des professes n'ont pas telle-

41. Quelques cas nous sont connus: «Mademoiselle Lemieux s'appelle maintenant Sœur Marie-Ignace. C'est le nom du "saint évêque » [Ignace Bourget] qu'apprécie et vénère la mère de la nouvelle novice.» Germaine Duval snjm, Par le chemin du Roi..., op. cit., 202. Le prénom de sœur Gérard Marie proviendrait de l'évêque ayant célébré sa prise de voile. La religieuse semble avoir été surprise par ce choix puisque ce nom n'était porté ni par son père ni par ses frères. 
ment de souvenirs à ce sujet. Les élèves s'adressaient à elles avec l'expression «ma sœur». Certains noms ont bien pu susciter sourires et rires, mais les enfants se sont bien gardés de les exprimer devant les SNJM.

\section{CONCLUSION}

Cette étude sur la dénomination chez les Sours des SNJM entre 1844 et 1969 fait ressortir un certain nombre de constatations. Il appert d'abord que la communauté, comme plusieurs autres, ne possédait pas de politique écrite concernant l'attribution d'un prénom masculin, mais que $39 \%$ des sœurs utilisaient totalement ou partiellement un prénom masculin. Un choix de prénoms était soumis par la postulante; la secrétaire générale, seule ou en consultation, en faisait l'attribution. Les noms choisis respectaient la liste des saints et des saintes approuvée par l'Église catholique. Par ailleurs, la dénomination en usage dans la communauté se distingue à maints égards de son équivalent civil. Toutes les professes recevaient le prénom Marie mais son usage courant variait. Pour certaines, il existait comme prénom simple ou comme une partie d'un prénom composé. Pour d'autres, il n'était qu'un élément symbolique précédant un prénom usuel simple accompagné d'un suffixe religieux ou d'un prénom composé entièrement masculin ou féminin. L'association des différents éléments provoquait parfois des juxtapositions insolites. Parmi ces prénoms composés, on constate une nette domination des prénoms entièrement féminins. Dans les prénoms masculins, "Jean» se détache particulièrement. Il n’apparaît pourtant pas, pour la même période, parmi les prénoms les plus populaires chez les garçons, dans la société civile. Le prénom du père, bien que visible chez plus de $15 \%$ des professes, n'occupe pas une place importante. En fait, en comparant avec d'autres congrégations et en analysant les prénoms attribués chez les SNJM, le constat qui s'impose est que l'attribution des noms d'hommes est relativement courante au début du $\mathrm{xx}^{\mathrm{e}}$ siècle, mais qu'elle n'est pas généralisée. En d'autres mots, l'usage des prénoms masculins est là comme une tradition dont les origines restent floues. Les membres du Conseil, qui avaient le dernier mot, ont préféré construire une identité collective féminine forte, et ce, sans soumettre la majorité des membres à une dénomination individuelle masculine.

Ces données nuancent donc, en partie, la vision d'une masculinisation, d'une indifférenciation volontaire des religieuses par leur prénom. Dans les rapports quotidiens, plus de $79 \%$ des religieuses s'identifient totalement ou partiellement avec un prénom féminin. Chez les SNJM, d'après les entrevues effectuées, le prénom de la professe n'a jamais eu d'impact 
sur sa carrière. De plus, rien ne dit que l'attribution de ces prénoms se soit développée comme réponse féministe à une subordination à la fois sociale et professionnelle. L'insistance du discours officiel de l'Église sur la différence traditionnelle entre les hommes et les femmes, «légitime une hiérarchisation des sexes et maintient l'appropriation masculine du sacré et accentue l'occultation des femmes ${ }^{42}$ ", mais pas sur le plan de la prénomination. De nouvelles études pourraient tenter de saisir comment la population percevait cette pratique. On pourrait aussi élargir le bassin des références masculines au-delà du père afin de saisir, par exemple, l'influence des frères ${ }^{43}$, ou de certains clercs. D'autres analyses pourraient pousser la comparaison entre différentes congrégations, afin de dégager des similitudes et des différences dans le temps et l'espace. Ultimement, il faut peut-être s'attarder aux communautés nées sous l'épiscopat de $\mathrm{M}^{\mathrm{gr}}$ Bourget, qui nous apparaissent plus touchées par ce phénomène. L'étude de ces femmes, à la frontière de l'univers laïque et de celui du clergé masculin, mérite encore notre attention.

42. Denise Veillette, "Hiérarchisation sociale des sexes...», loc. cit., 17.

43. Une petite enquête réalisée par les responsables des archives des SNJM, sur un groupe témoin de 22 sœurs vivantes, a permis de découvrir que 7 d'entre elles avaient un nom religieux d'homme. Sur ces 7 noms, 6 étaient le nom d'un frère et non celui du père. Le $7^{\mathrm{e}}$ nom était celui d'un saint récemment canonisé. Pour faire une telle recherche sur le nom du frère, il faudrait consulter la fiche personnelle de chaque sœur. 